\title{
Transient partial response of poorly-differentiated thyroid carcinoma to sunitinib treatment: A case report
}

\author{
LI JIN DUO, JIANG RONG, WANG BIN, MA CHUN HUA, SUN LI WEI and LV YUAN \\ Department of Tumor Intervention, Tianjin Key Laboratory of Cerebral Vascular and Neurodegenerative Diseases, \\ Tianjin Huanhu Hospital, Tianjin 300060, P.R. China
}

Received June 26, 2014; Accepted March 3, 2015

DOI: 10.3892/ol.2015.3174

\begin{abstract}
Thyroid cancer is the most common endocrine malignancy, with an increasing prevalence worldwide. Poorly-differentiated thyroid cancer (PDTC) is relatively rare and its prognosis is poor. To date, no ideal treatment strategy is available for patients with advanced recurrent PDTC, particularly for patients in crisis. However, partial success in treating thyroid cancer has been achieved with targeted therapy, and advances made in understanding the molecular biology of the tumor. The current study describes the case of a patient diagnosed with PDTC following presentation with hoarseness, orthopnea, and a large right neck mass. A transient partial response to sunitinib malate treatment was achieved for $>3$ months. In addition, the current study reviewed the relevant literature and discussed the therapeutic value of sunitinib as a more favorable treatment strategy for patients with advanced recurrent PDTC compared with the currently available treatments. Successful treatment with sunitinib, as well as molecular analysis of the tumor, occurred in the present case. Sunitinib was determined to have potential in treating thyroid tumors, however, larger prospective studies are required to validate the findings of the current case study prior to the application of this agent in clinical practice.
\end{abstract}

\section{Introduction}

Thyroid cancer is the most common endocrine malignancy (1), with an increasing incidence rate worldwide (2). Differentiated thyroid cancer (DTC) accounts for $80-90 \%$ of all cases of thyroid cancer and is primarily responsible for the increased incidence of this disease (3). By contrast, poorly-differentiated thyroid cancer (PDTC) represents only 5\% of all cases of

Correspondence to: Dr Jiang Rong, Department of Tumor Intervention, Tianjin Key Laboratory of Cerebral Vascular and Neurodegenerative Diseases, Tianjin Huanhu Hospital, 122 Meteorological Station Road, Tianjin 300060, P.R. China E-mail: jiangrong2003@126.com

Key words: sunitinib, thyroid carcinoma, poorly-differentiated, crisis thyroid cancer cases (4); however, PDTC is more aggressive and is associated with worse outcomes compared with DTC. For patients with advanced DTC that is not suitable for radioiodine therapy, surgical resection or local therapy with external-beam radiation, there are only a limited number of therapeutic strategies available (5).

One-third of all patients with PDTC develop recurrent disease, and only $30 \%$ of patients with distant metastases may achieve complete remission with radioiodine therapy $(6,7)$. As recurrent PDTC is aggressive and often associated with poor outcomes, these patients would primarily benefit from the development of novel therapeutic strategies (8). Recent studies have revealed information regarding the biological and molecular mechanisms underlying thyroid cancer, facilitating the identification of novel molecular targets for the treatment of the patients with PDTC. At present, the use of suntinib for the treatment of thyroid cancers is under investigation (ClinicalTrials.gov identifiers, NCT00510640, NCT00381641 and NCT00668811), however, there are very few reports evaluating sunitinib for PDTC.

The present study describes a case of recurrent PDTC that was not amenable to any currently available therapeutic strategies, but was successfully treated with sunitinib. Written informed consent for this case report was obtained from the patient's family.

\section{Case report}

A 49-year-old female was referred to Tianjin Huanhu Hospital (Tianjin, China) in October 2013 due to the post-operative recurrence of PDTC and lung metastases.

The patient was initially admitted to the Affiliated Hospital of Inner Mongolia Medical College (Hohhot, Mongolia) in September 2012 with neck masses. The patient underwent resection of the right lobe of the thyroid and isthmus, as well as subtotal resection of the left lobe. A pathological examination revealed that the $40 \times 30 \times 40-\mathrm{mm}$ tumor in the right lobe was a poorly-differentiated papillary thyroid carcinoma with partial neuroendocrine large-cell undifferentiated carcinoma and Hashimoto's thyroiditis, a type of PDTC. The tumor was immunoreactive to thyroid transcription factor 1, thyroglobulin and cytokeratin 19, with a Ki-67 labeling index of $<70 \%$. Otherwise, the left thyroid lobe and isthmus lesions were benign. The patient opted for treatment with herbal and 

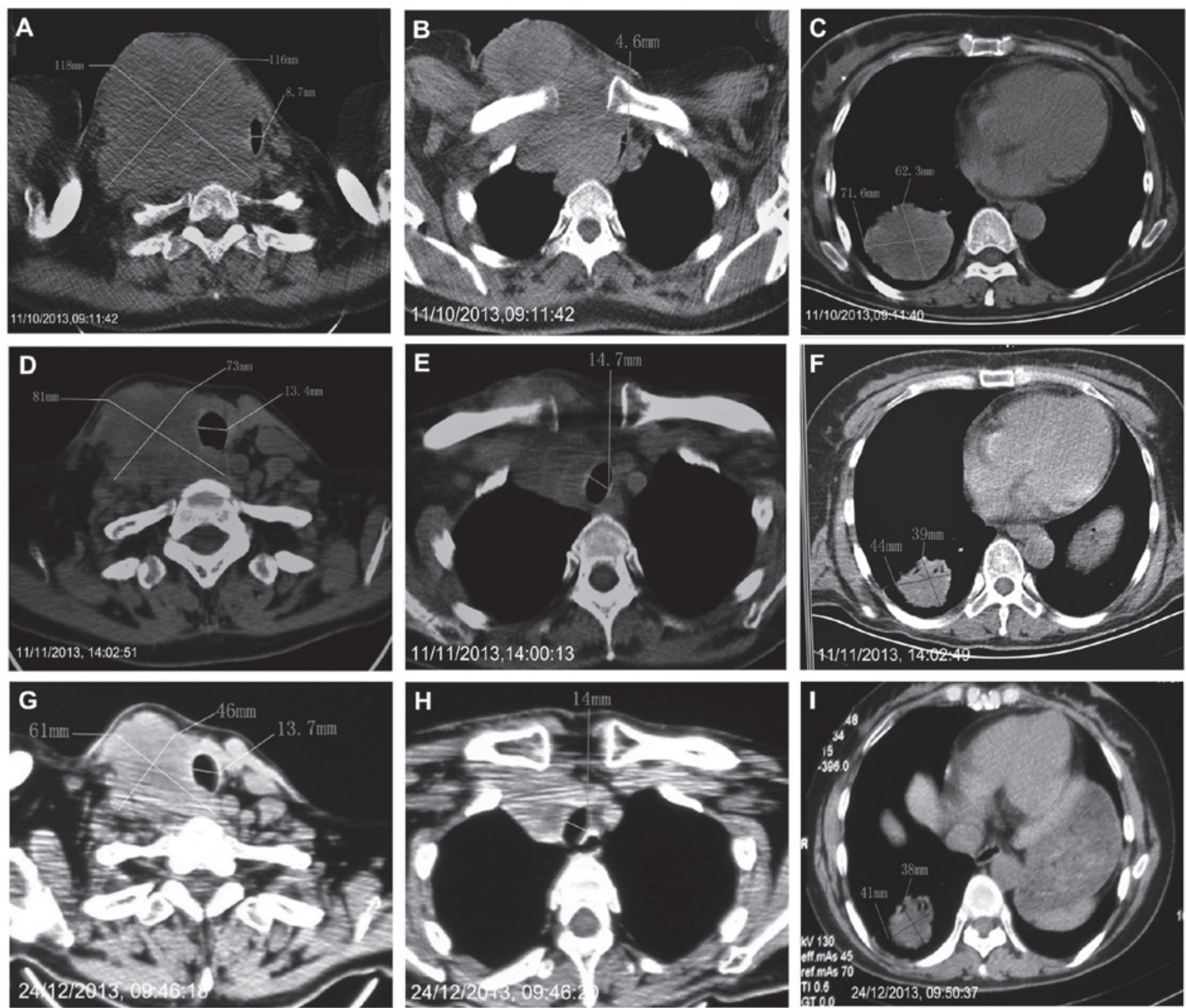

Figure 1. Computed tomography scans demonstrating an objective response according to the response evaluation criteria in solid tumors. (A-C) Prior to the initiation of therapy, a large mass with uneven density in the right lobe of the thyroid was observed. The trachea was compressed to the left in the neck. A right supraclavicular conglomerate of lymph nodes was detected and multiple enlarged lymph nodes were compressed in the tracheoesophageal groove and the right paratracheal space. Additionally, multiple metastases were present in the lungs. (D-F) After 4 weeks of therapy, the mass in the right lobe of the thyroid and conglomerated lymph nodes were reduced, and the tracheal compression had improved. (G-I) After 10 weeks of therapy, the mass in the right lobe of the thyroid and conglomerated lymph nodes had further reduced, and the compressed trachea continued to improve.

conservative traditional Chinese medicine alone (drug name and dose unknown), which was administered by a family doctor, as opposed to radiotherapy and/or chemotherapy due to the patient's concerns regarding possible side-effects. Two and a half months after surgery, fluorine-18 fluorodeoxyglucose positron emission tomography/computed tomography (CT) revealed tumor recurrence in the resection site and metastases in the posterior basal segment of the lower lobe of the right lung. However, the patient insisted on the continuation of traditional Chinese medicine. The right neck mass became ulcerous in August 2013, with dyspnea appearing one month later and gradually worsening. The patient was eventually admitted to Tianjin Huanhu Hospital for further diagnosis and treatment. Despite weight loss of $10 \mathrm{~kg}$ over two months, the patient's disposition, diet and overall physical condition were adequate. In additional to traditional Chinese medicine, the patient had been taking thyroid tablets (dose, $120 \mathrm{mg}$ daily) for hypothyroidism since undergoing surgery. Furthermore, the patient's diabetes had been managed with routine medication for three years, and no other medical problems or family history of malignancy were noted.

The patient's voice sounded hoarse with orthopnea upon physical examination. A large right neck mass was clearly visible, measuring $10 \times 10 \mathrm{~cm}$, and an area of skin ulceration of $\sim 5 \times 5 \mathrm{~cm}$ was present on the surface in the middle of the mass. A decrease in respiratory sounds was found for each lung and edema was notable in the right upper limb.

A CT scan revealed a large mass with uneven density in the right lobe of the thyroid. The trachea was compressed to the left in the neck, with CT indicating a right supraclavicular conglomerate of lymph nodes and multiple enlarged lymph nodes compressed in the tracheoesophageal groove and the right paratracheal space. Additionally, multiple metastases were present in the lungs (Fig. 1).

The patient experienced dyspnea and the inability to lie down. The local recurrence that developed following tumor resection was considered to be unresectable, therefore, the 


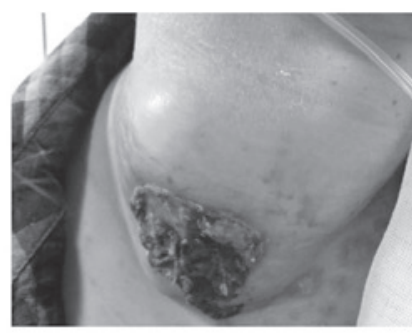

$17 / 10 / 2013$

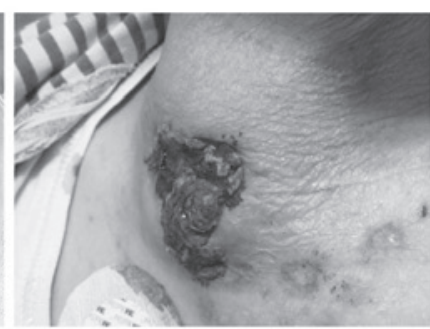

$25 / 10 / 2013$

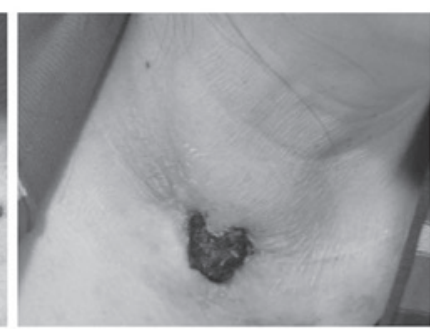

$11 / 11 / 2013$

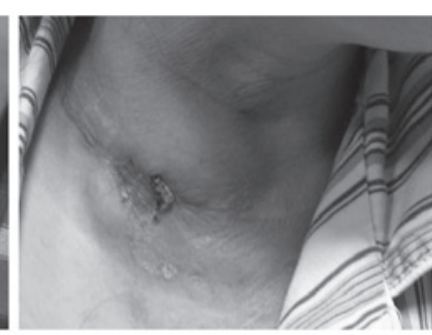

$25 / 11 / 2013$

Figure 2. Macroscopic images of the poorly-differentiated thyroid neck tumor over time.

patient was selected to undergo treatment with sunitinib. Following approval by the Institutional Review Board of Tianjin Huanhu Hospital and receipt of written informed consent from the patient, sunitinib malate (Sutent ${ }^{\circledR}$; Pfizer, Inc., New York, NY, USA) treatment commenced in October 2013 at a dose of $37.5 \mathrm{mg} /$ day.

After only seven days of treatment, the dyspnea symptoms improved and the patient was able to sleep in a semi-supine position. The dyspnea completely disappeared one month later. A lasting response was observed over three months of therapy, with sunitinib successfully inducing the shrinkage of the large right neck mass and lung metastases (Figs. 1 and 2). CT indicated evidence of an objective response, according to the response evaluation criteria in solid tumors (RECIST) (9), indicating that the current patient exhibited a partial response to treatment with sunitinib (Fig. 1). During treatment, the patient experienced mild fatigue and the development of hand/foot syndrome was unavoidable. Grade III leukopenia was observed one month after therapy commenced, resulting in the temporary discontinuation of treatment for four weeks. At the end of January 2014, grade III thrombocytopenia was observed, thus, sunitinib treatment was terminated. The tumor eventually progressed and the patient succumbed to the disease in February 2014. The time to progression was 3.25 months.

\section{Discussion}

Primary thyroid cancer presents as one of four histological subtypes: Papillary thyroid cancer (PTC), follicular thyroid cancer (FTC), medullary thyroid cancer (MTC) and anaplastic thyroid cancer (ATC), with PTC and FTC typically grouped together as DTC. DTC is conventionally treated using a combined approach of total thyroidectomy and radioactive iodine (RAI) therapy prior to thyroid-stimulating hormone suppression. Such an approach generally results in a good prognosis for patients with DTC, with a five-year disease-associated survival rate of $97.8 \%$ (2).

Despite the good initial outcome of surgery for the vast majority of patients, $10-15 \%$ experience recurrent disease, with $\sim 5 \%$ exhibiting distant metastases at presentation (10). The clinical management of patients with recurrent and advanced disease is often challenging. Their response to conventional chemotherapy is typically poor, with commonly used cytostatic chemotherapeutic agents resulting in limited and transient response rates of 0-22\%, and typically causing significant toxicity (11). In certain cases, repeated surgery may control locoregional disease, however, it is not a viable option in cases of anaplastic cancer, for which possible treatment strategies are limited due to almost universally poor outcomes (12). The tumor pathology of the current patient was complex, consisting of poorly-differentiated papillary thyroid carcinoma combined with partial neuroendocrine large-cell undifferentiated carcinoma. Due to the possibly difficult complete resection of the tumor and the patient's orthopnea, a second surgical procedure, RAI, external-beam radiation or chemotherapy were not ideal treatment strategies.

The management of patients with PDTC is challenging and controversial, with no effective treatment strategies available prior to the recent development of multi-targeted tyrosine kinase inhibitors (13). Recent advances in the understanding of the biology of thyroid cancer, including DTC, MTC and ATC, have enabled the identification of various important molecular changes in thyroid cancer cells. These changes provide the basis for developing targeted therapies that have been considered for the treatment of thyroid cancer (12).

Various diagnostic and prognostic molecular markers, including $B R A F$ and $R A S$ point mutations, $R E T / P T C$ and $P A X 8 / P P A R \gamma$ gene rearrangements, mitogen-activated protein kinase (MAPK), phosphoinositide 3-kinase (PI3K), tumor protein $\mathrm{p} 53, \mathrm{Wnt}-\beta$ catenin, hypoxia-inducible factor $1 \alpha$ and nuclear factor- $\kappa \mathrm{B}$ signaling pathways, microRNA profiles, and aberrant methylation, have been identified in $>70 \%$ of DTC cases (14). Ras/Raf/MEK/ERK (MAPK) and $\mathrm{PI} 3 \mathrm{~K} / \mathrm{Akt} / \mathrm{mammalian}$ target of rapamycin are the two major signaling pathways indicated to be involved in thyroid cancer, and are closely associated with the promotion of cell survival, cell cycle progression, migration, proliferation, metabolism, tumorigenesis and angiogenesis (15). Furthermore, the most frequently observed nucleotide substitutions in DTC involve $B R A F, R A S(H-, N$ - and $K-R A S)$ and RET $(16,17)$.

$B R A F$, an important regulator of thyroid protein expression and cell proliferation, and the most potent activator of the MAPK pathway, is commonly mutated in thyroid cancer. Previously, the presence of $B R A F$ V600E has been associated with a higher prevalence of persistent and recurrent disease (18). As $B R A F$ has been indicated to be involved in PTC and ATC, it is the focus of various studies regarding the development of targeted therapies for thyroid cancer (12). In addition, $B R A F$ is associated with the overexpression of vascular endothelial growth factor (VEGF), a protein with a role in angiogenesis. VEGF and VEGF receptor (VEGFR), as well as various growth factors, including platelet-derived growth factor (PDGF) and fibroblast growth factor, are 
often overexpressed in thyroid cancer cells, constituting a second group of possible targets for the treatment of thyroid cancer (12).

The Ras family of small GTPases is composed of K-, $\mathrm{H}$ - and N-Ras. These G-proteins are fundamental in the transduction of intracellular signals from the cell membrane, however, constitutive activation of these three genes has been observed in all thyroid tumors originating from follicular cells (FTC). Furthermore, RAS substitutions are frequently detected in ATC and PDTCs. The most commonly reported $R A S$ mutations in thyroid tumors are those in codon 61 of $N$-RAS and in $H$-RAS, however, others have been described $(19,20)$.

$R E T$ is a proto-oncogene that encodes a transmembrane tyrosine-kinase receptor. The gene is located on chromosome $10 \mathrm{q} 11.2$ and its activation stimulates various signaling pathways, including the MAPK cascade. RET is not typically expressed in the follicular cells of the thyroid gland, however, germline point mutations of RET have been identified in $\sim 98 \%$ of all hereditary MTC cases and somatic RET mutations are present in $\sim 40 \%$ (range, $30-70 \%$ ) of sporadic cases (21).

In the current case, $R A S, B R A F$ and $R E T$ mutation status was unavailable, as the patient did not wish to undergo tumor biopsies. We propose that these genes should be routinely surveyed in future cases for individualized therapy.

Sunitinib is an oral tyrosine kinase inhibitor that targets VEGFR-1, -2 and -3, RET, KIT, PDGF receptors $\alpha$ and $\beta$, FMS-like tyrosine kinase 3 and colony-stimulating factor receptor type 1 (22). In an initial open-label, phase II study (23), 43 patients with metastatic, iodine-refractory thyroid carcinoma of all histological subtypes (37 DTCs and 6 MTCs) exhibited biochemical and objective RECIST responses to six-week cycles (four weeks on and two weeks off) of sunitinib at a single daily dose of $50 \mathrm{mg}$. Of the 31 patients with DTC who completed two cycles of sunitinib therapy, $13 \%$ exhibited a partial response, $68 \%$ experienced stable disease, $10 \%$ experienced progressive disease and $9 \%$ were not evaluable. Thus, the objective response rate was $13 \%$ and the disease control rate was $81 \%$ (23). A second phase II trial (24) of sunitinib (37.5 $\mathrm{mg}$ daily) in patients with progressive DTC $(n=28)$ or MTC $(n=7)$ reported a complete response in $3 \%$ of patients, a partial response in $28 \%$ of patients and stable disease in $46 \%$ of patients. Toxicities included fatigue, neutropenia, hand-foot syndrome, diarrhea and leukopenia. In addition, one patient who was administered with anticoagulation medication succumbed due to gastrointestinal bleeding (24). A third open-label, phase II trial (ClinicalTrials.gov identifier, NCT00510640) has been completed, however, the study results have yet to be published. Furthermore, no phase III trial of sunitinib for the treatment of thyroid cancer has been conducted thus far.

In the present case, treatment with sunitinib resulted in a significant reduction in tumor size and caused symptom amelioration. A noticeable response was observed in the neck tumor, potentially providing opportunities for additional treatments. Therefore, the current study indicates that sunitinib may be a good treatment strategy for similar cases of PDTC. However, genetic testing should be considered for more individualized therapy and attention should be focused on possible adverse reactions, particularly hematological toxicity.

\section{Acknowledgements}

The authors would like to thank Dr Li Xiao Long, Department of Head and Neck Tumors at Tianjin Medical University Cancer Institute and Hospital (Tianjin, China), for providing technical assistance. The authors would also like to thank Dr $\mathrm{Fu}$ Le Jun from the Imaging Department at Tianjin Huanhu Hospital (Tianjin, China) for preparing the tumor images.

\section{References}

1. Agate L, Lorusso L and Elisei R: New and old knowledge on differentiated thyroid cancer epidemiology and risk factors. J Endocrinol Invest 35 (Suppl): 3-9, 2012.

2. National Cancer Institute: Surveillance Epidemiology and End Results Program: SEER stat fact sheets: Thyroid cancer. http://seer.cancer.gov/statfacts/html/thyro.html\#incidence-mortality. Accessed, 15th June 2013.

3. Cooper DS, Doherty GM, Haugen BR, et al; American Thyroid Association (ATA) Guidelines Taskforce on Thyroid Nodules and Differentiated Thyroid Cancer: Revised American Thyroid Association management guidelines for patients with thyroid nodules and differentiated thyroid cancer. Thyroid 19: 1167-1214, 2009.

4. Brose M, Nutting C, Jarzab B, et al: Sorafenib in locally advanced or metastatic patients with radioactive iodine-refractory differentiated thyroid cancer: The phase III DECISION trial. J Clin Oncol 31 (Suppl): Abstract 4, 2013.

5. DeSantis CE, Lin CC, Mariotto AB, et al: Cancer treatment and survivorship statistics, 2014. CA Cancer J Clin 64: 252-271, 2014.

6. Dohán O, De la Vieja A, Paroder V, et al: The sodium/iodide symporter (NIS): characterization, regulation and medical significance. Endocr Rev 24: 48-77, 2003.

7. Schlumberger MJ: Diagnostic follow-up of well-differentiated thyroid carcinoma: historical perspective and current status. J Endocrinol Invest 22 (Suppl): 3-7, 1999.

8. Durante C, Haddy N, Baudin E, et al: Long-term outcome of 444 patients with distant metastases from papillary and follicular thyroid carcinoma: benefits and limits of radioiodine therapy. J Clin Endocrinol Metab 91: 2892-2899, 2006.

9. Eisenhauer EA, Therasse P, Bogaerts J, et al: New response evaluation criteria in solid tumours: revised RECIST guideline (version 1.1). Eur J Cancer 45: 228-247, 2009.

10. Perez CA, Santos ES, Arango BA, Raez LE and Cohen EE: Novel molecular targeted therapies for refractory thyroid cancer. Head Neck 34: 736-745, 2012.

11. Baudin E and Schlumberger M: New therapeutic approaches for metastatic thyroid carcinoma. Lancet Oncol 8: 148-156, 2007.

12. Nixon IJ, Shaha AR and Tuttle MR: Targeted therapy in thyroid cancer. Curr Opin Otolaryngol Head Neck Surg 21: 130-134, 2013.

13. Antonelli A, Fallahi P, Ferrari SM, et al: New targeted therapies for thyroid cancer. Curr Genomics 12: 626-631, 2011.

14. Omur O and Baran Y: An update on molecular biology of thyroid cancers. Crit Rev Oncol Hematol 90: 233-252, 2014.

15. Barollo S, Bertazza L, Baldini E, et al: The combination of RAF265, SB590885, ZSTK474 on thyroid cancer cell lines deeply impact on proliferation and MAPK and PI3K/Akt signaling pathways. Invest New Drugs 32: 626-635, 2014.

16. Patel KN and Shaha AR: Poorly differentiated thyroid cancer. Curr Opin Otolaryngol Head Neck Surg 22: 121-126, 2014.

17. Sherman SI: Targeted therapy of thyroid cancer. Biochem Pharmacol 80: 592-601, 2010.

18. Kebebew E, Weng J, Bauer J, et al: The prevalence and prognostic value of BRAF mutation in thyroid cancer. Ann Surg 246: 466-470, discussion 470-461, 2007.

19. Nikiforova MN, Lynch RA, Biddinger PW, et al: RAS point mutations and PAX8-PPAR gamma rearrangement in thyroid tumors: evidence for distinct molecular pathways in thyroid follicular carcinoma. J Clin Endocrinol Metab 88: 2318-2326, 2003.

20. Vasko V, Ferrand M, Di Cristofaro J, Carayon P, Henry JF and de Micco C. Specific pattern of RAS oncogene mutations in follicular thyroid tumors. J Clin Endocrinol Metab 88: 2745-2752, 2003. 
21. Castellone MD and Santoro M: Dysregulated RET signaling in thyroid cancer. Endocrinol Metab Clin North Am 37: 363-374, 2008

22. Sherman SI: Early clinical studies of novel therapies for thyroid cancers. Endocrinol Metab Clin North Am 37: 511-524, 2008.

23. Cohen EE, Needles BM, Cullen KJ, et al: Phase 2 study of sunitinib in refractory thyroid cancer. ASCO Meeting Abstracts 26: 6025, 2008.
24. Carr LL, Mankoff DA, Goulart BH, et al: Phase II study of daily sunitinib in FDG-PET-positive, iodine-refractory differentiated thyroid cancer and metastatic medullary carcinoma of the thyroid with functional imaging correlation. Clin Cancer Res 16: 5260-5268, 2010 